Manju Rajaram, Adimulam Ganga Ravindra, Dharm Prakash Dwivedi, Jayalakshmi Ramakrishnan, Palanivel Chinnakali, Madhusmita Mohanty Mohapatra

Jawaharlal Institute of Postgraduate Medical Education and Research, Puducherry, India

\title{
HIV status among presumptive tuberculosis cases attending tertiary care centre in South India
}

\author{
HIV status among presumptive tuberculosis cases
}

\begin{abstract}
Introduction: In India, to increase human immunodeficiency virus (HIV) case detection, the National Technical Working Group on TB /HIV had made it mandatory to screen for HIV infection among presumptive tuberculosis (TB) cases.

Material and methods: Our study was a cross-sectional study, conducted between June 2015 and December 2016 in a tertiary care institute (JIPMER), in South India, to estimate the prevalence of HIV among presumptive TB cases.

Results: Among the 964 presumptive TB cases who attended pulmonary medicine OPD, 189 patients were sputum acid-fast bacilli (AFB) positive. Among the 189 sputum positive cases, 9 were HIV positive. Of the 964 presumptive TB cases, 879 gave consent for HIV testing and 33 (3.7\%) turned out to be HIV positive. If only sputum positive cases had been screened for HIV, we would have missed 24 new HIV positive cases. The number needed to screen was 27 among presumptive TB cases and 18 among TB patients.

Conclusions: The uptake of HIV testing (91\%) and the diagnostic yield of $3.7 \%$ of HIV positive cases among the presumptive TB patients is quite high compared to that of the Revised National Tuberculosis Control Programme (RNTCP) mechanism of Puducherry. This reinforces the need to screen all the presumptive cases for HIV infection, to increase HIV case detection so that anti-retroviral therapy (ART) can be initiated early.
\end{abstract}

Key words: presumptive TB cases, HIV testing, Provider-Initiated HIV Testing and Counseling, sputum positive cases, co-infection

Adv Respir Med. 2018; 86: 211-214

\section{Introduction}

In India, around $4 \%$ of tuberculosis (TB) patients are estimated to be HIV co-infected [1]. In patients being treated for TB, HIV co-infection increases the morbidity and mortality. In these patients, many a time, HIV is undiagnosed or diagnosed very late, leading to delay in initiation of anti-retroviral therapy (ART) for cases which merit treatment with ART.

For early diagnosis of HIV infection among patients presenting to the health care settings with symptoms of tuberculosis, the World Health Organization (WHO) had issued an updated policy statement in March 2012 that Provider- Initiated HIV Testing and Counseling (PITC) should be offered not only to TB patients but also to all presumptive TB cases [2]. This recommendation had been given basing on the studies in sub-Saharan African countries, which had shown very high HIV prevalence among presumptive TB cases, sometimes even higher than HIV prevalence among TB patients [3, 4].

Considering the country evidence [1] and global recommendation, the National Technical Working Group on TB/HIV decided to implement PITC among presumptive TB cases in all high HIV prevalent settings in India (A and B category districts) in a phased manner. Routine screening of presumptive TB cases for HIV is being implemented in phase wise manner throughout the country.

In India, all diagnosed TB patients are being screened for HIV infection. However, in spite of the recommendations, the screening for HIV infection among presumptive TB cases has been suboptimal. Hence this study was undertaken in

Address for correspondence: Manju Rajaram, JIPMER, Dhanvantari Nagar, 605006 Puducherry, India, e-mail: mail2manju22@gmail.com

DOI: 10.5603/ARM.2018.0033

Received: 27.06.2018

Copyright (C) 2018 PTChP

ISSN 2451-4934 
JIPMER, a tertiary care institute in Puducherry, South India, to estimate the prevalence of HIV in presumptive TB cases, as compared to TB patients.

\section{Material and methods}

This was a hospital-based cross-sectional descriptive study conducted among patients attending a tertiary care research institute in South India. Approximately 1600 patients attend pulmonary medicine Out-Patient Department (OPD) in a month. All patients aged more than 14 years who had attended pulmonary medicine OPD in between June 2015 and December 2016, with symptoms suggestive of tuberculosis (presumptive TB) were taken up for the study. Presumptive pulmonary TB refers to a person with any of the symptoms and signs suggestive of TB including a cough for more than 2 weeks, fever for more than 2 weeks, significant weight loss, hemoptysis, any abnormality in the chest radiograph.

After informed consent, details like demographic characteristics, history of the presenting complaints, personal history and past history of tuberculosis were taken from all patients, using pre-structured proforma. All the presumptive TB patients were requested to give blood sample for HIV testing. After taking informed consent, HIV testing was done as per the National AIDS Control Organization (NACO) guidelines [5]. Blood samples were subjected to following rapid diagnostic tests - MERISCREEN HIV 1-2 WB test, Signal HIV Flow through HIV 1+2 Spot Immunodot test, COMBAIDS HIV 1+2 Immunodot test. A positive reaction in all three tests was necessary for a patient to be diagnosed as HIV positive.
All categorical variables such as age group, gender, clinical characteristics, sputum smear results, and HIV testing status and results were expressed as frequency and percentages.

\section{Results}

Of the 1089 patients who had presumptive tuberculosis symptoms, 964 patients gave two sputum samples (spot and early morning) for AFB smear examination. Among the 964 presumptive TB patients, 189 were diagnosed to be sputum positive.

More than $80 \%$ of the cases were in the age group between 25 and 64 years. Approximately three-fourths of the presumptive TB and pulmonary TB was in the age group between 35 and 64 years. Similarly, men constituted a greater proportion of presumptive TB and sputum positive TB compared to women (Table 1). A cough for more than 2 weeks was the most common presentation found among the presumptive TB patients (Fig. 1). Low-grade fever (23.9\%), weight loss $(13.1 \%)$ and loss of appetite (15.9\%) were other common presentation found in the study. Almost $52.4 \%$ of the sputum positive TB cases had smoking habits.

\section{HIV status among presumptive pulmonary tuberculosis patients}

Out of 964 presumptive TB cases who gave 2 sputum samples for AFB smear examination, 879 (91\%) cases gave consent for checking HIV status. Out of 879 presumptive TB cases, 33 (3.7\%) turned out to be HIV positive. All the 33 cases were newly diagnosed. Out of the 33 HIV positive cases, 24 were sputum AFB smear-negative (Table 2).

Table 1. Age and gender distribution of presumptive TB and sputum positive PTB cases

\begin{tabular}{lccc}
\hline Age group(years) & $\begin{array}{c}\text { Presumptive tuberculosis } \\
\text { patients } \mathbf{n}=\mathbf{9 6 4}(\%)\end{array}$ & $\begin{array}{c}\text { Sputum positive cases } \\
\text { (n = 189) }\end{array}$ & $\begin{array}{c}\text { Percentage of sputum positive } \\
\text { cases }\end{array}$ \\
\hline $15-24$ & $134(13.9)$ & 20 & 10.6 \\
$25-34$ & $113(11.7)$ & 22 & 11.6 \\
$35-44$ & $208(21.6)$ & 52 & 27.5 \\
$45-54$ & $239(24.7)$ & 55 & 29.1 \\
$55-64$ & $176(18.25)$ & 32 & 16.9 \\
Above 65 & $94(9.75)$ & 8 & 4.2 \\
Total & 964 & 189 & 100 \\
Gender & & & 79.4 \\
Male & $603(62.55)$ & 150 & 20.6 \\
Female & $361(37.44)$ & 39 & 100 \\
Total & 964 & 189 & \\
\hline
\end{tabular}




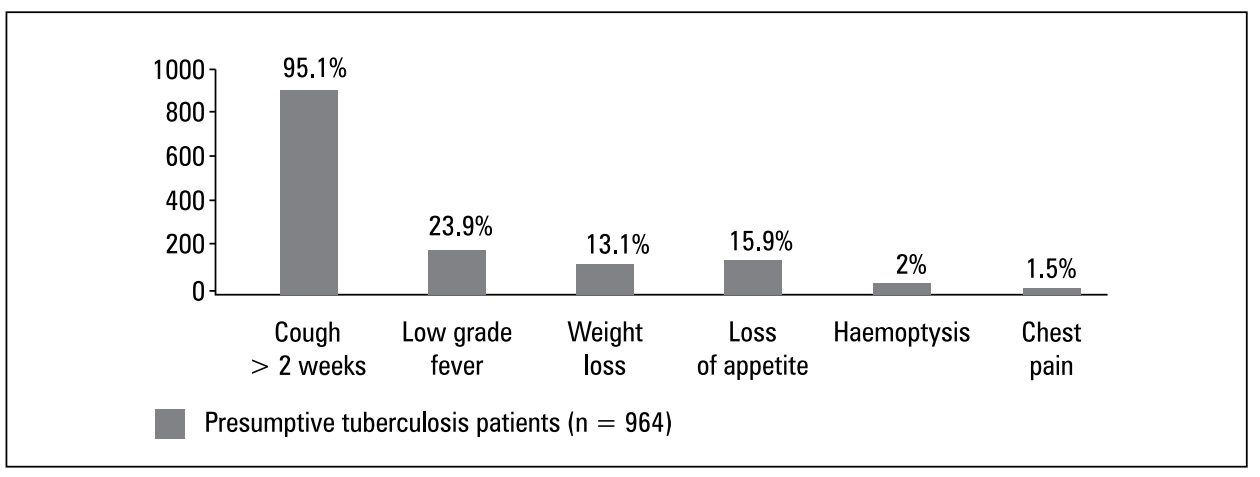

Figure 1. Clinical presentation of presumptive tuberculosis patient $(n=964)$

Table 2. HIV positivity in presumptive TB cases

\begin{tabular}{|c|c|c|c|c|}
\hline & $\begin{array}{l}\text { No. of cases } \\
\text { who gave consent } \\
\text { for HIV testing }\end{array}$ & $\begin{array}{l}\text { No. of HIV positive } \\
\text { cases }\end{array}$ & Prevalence of HIV & $\begin{array}{l}\text { The number needed } \\
\text { to screen (NNS) }\end{array}$ \\
\hline $\begin{array}{l}\text { Presumptive TB cases } \\
(\mathrm{n}=964)\end{array}$ & $879(91 \%)$ & 33 & $3.7 \%$ & 27 \\
\hline $\begin{array}{l}\text { Sputum AFB smear-positive } \\
\text { cases }(n=189)\end{array}$ & $163(86 \%)$ & 9 & $5.5 \%$ & 18 \\
\hline
\end{tabular}

Out of 189 sputum positive cases of pulmonary tuberculosis, $163(86 \%)$ subjects gave consent for checking HIV status. Out of the 163 sputum positive cases, 9 (5.5\%) were HIV positive. Therefore, 24 extra HIV positive cases have been detected by testing presumptive TB patients. The number needed to screen (NNS) to find an additional HIV case was found to be 27 among presumptive cases and 18 among sputum smear-positive TB patients (Table 2).

\section{Discussion and conclusions}

In our study, out of a total of 33 newly diagnosed HIV cases obtained by screening 879 presumptive TB patients who gave consent for HIV testing, 24 (72.7\%) were AFB smear negative. Had only confirmed TB cases (sputum AFB positive) been screened for HIV infection, we would have diagnosed only 9 new HIV positive cases and missed 24 cases. Hence we can reasonably state that if intensified efforts are made to screen the population of presumptive TB patients, additional new HIV cases could be diagnosed in the community and initiated on antiretroviral therapy (ART) if found to be eligible. Also, these cases could be initiated on isoniazid prophylactic therapy (IPT), which would go a long way in decreasing the occurrence of TB infection in HIV positive cases.
Also, in our study, the prevalence of HIV among the presumptive TB cases was $3.7 \%$. This is comparable to the study by Palanivel et al. [6] in 2013 in Puducherry, wherein the prevalence of HIV among presumptive TB cases was $3.3 \%$. However, it was a retrospective record-based study. In a study done in Karnataka by Kumar et al [7] in 2012, 12\% of presumptive TB patients were found to be HIV positive.

In our study, 91\% (879 out of 964) of the presumptive TB cases could be screened for HIV. In the Union territory of Puducherry, on average, only $35-40 \%$ of presumptive TB cases are being screened for HIV every year. This is due to the fact that these presumptive TB cases were recruited as part of the study and facilities were available for HIV testing in the tertiary care setting (JIPMER) in which the study was done. However, this may not be possible in many Designated Microscopic Centers wherein HIV testing may not be available. In our study, $3.7 \%$ of the presumptive TB cases turned out to be HIV positive and were hitherto undiagnosed cases. The yield is quite high as against the $0.5-0.7 \%$ of new cases being diagnosed every year by screening presumptive TB cases by the Revised National Tuberculosis Control Programme (RNTCP) mechanism in the Union territory of Puducherry. Hence, much more efforts are needed to increase HIV case detection among the presumptive TB cases in Puducherry. 
WHO and the Joint United Nations Programme on HIV/AIDS (UNIAIDS) have a vision of 90-90-90 strategy which emphasizes the need to detect $90 \%$ of all HIV infected patients in the community, treat $90 \%$ of those detected with anti-retroviral therapy and achieve viral suppression in $90 \%$ of those treated [8]. Screening of presumptive TB cases for HIV would definitely help us in achieving this vision.

The limitation of our study was that we do not have information about whether these HIV positive cases (among the presumptive TB population) were initiated on ART.

\section{Conclusion}

HIV testing among presumptive TB cases is quite a fruitful method of detecting new HIV cases in the community. High uptake of HIV testing among presumptive TB patients in a tertiary care setting indicates that it is feasible, and has enabled more than three times detection of HIV, which would have otherwise been missed because of sputum negativity. In A category district like Puducherry, where the prevalence of HIV is high, active screening for presumptive TB and HIV testing among them is all the more essential.

\section{Conflict of interest}

The authors declare no conflict of interest.

\section{References:}

1. India TB Report 2018. New Delhi: Central TB Division, Directorate General of Health Services, Ministry of Health and Family Welfare; 2018 Mar.

2. WHO policy on collaborative TB/HIV activities. Guidelines for national programmes and other stakeholders. World Health Organization; 2012.

3. Srikantiah P, Lin R, Walusimbi M, et al. Elevated HIV seroprevalence and risk behavior among Ugandan TB suspects: implications for HIV testing and prevention. Int J Tuberc Lung Dis. 2007; 11(2): 168-174, indexed in Pubmed: 17263287.

4. Deribew A, Negussu N, Melaku Z, et al. Investigation outcomes of tuberculosis suspects in the health centers of Addis Ababa, Ethiopia. PLoS One. 2011; 6(4): e18614, doi: 10.1371/journal. pone.0018614, indexed in Pubmed: 21526179.

5. National Guidelines for HIV Testing. National AIDS Control Organisation, Ministry of Health and Family Welfare; 2015.

6. Palanivel C, Kumar AMV, Mahalakshmi T, et al. Uptake of HIV testing and HIV positivity among presumptive tuberculosis patients at Puducherry, South India. Public Health Action. 2013; 3(3): 220-223, doi: 10.5588/pha.13.0045, indexed in Pubmed: 26393033.

7. Kumar AMv, Gupta D, Kumar A, et al. HIV Testing among Patients with Presumptive Tuberculosis: How Do We Implement in a Routine Programmatic Setting? Results of a Large Operational Research from India. PLoS One. 2016; 11(5): e0156487, doi: 10.1371/journal.pone.0156487, indexed in Pubmed: 27244055 .

8. 90-90-90 An ambitious treatment target to help end the AIDS epidemic. UNAIDS; 2014. 